\title{
Identification of Fifth-order Wiener and Hammerstein Channels based on the Estimation of an Associated Volterra Kernel
}

\author{
Zouhour Ben Ahmed \\ Laboratoire CEM \\ Sfax Engineering School, \\ BP 1173, 3038 Sfax, Tunisia
}

\author{
Gérard Favier \\ Laboratoire I3S \\ University of Nice Sophia Antipolis / CNRS \\ Les Algorithmes/Euclide B, 2000 route des Lucioles \\ 06900 Sophia Antipolis, France
}

\author{
Nabil Derbel \\ Laboratoire CEM \\ Sfax Engineering School, \\ BP 1173, 3038 Sfax, Tunisia
}

\begin{abstract}
In this paper, we consider the problem of identification of fifthorder Wiener and Hammerstein nonlinear communication channels using the estimation of an associated Volterra kernel. We exploit the special form of the fifth-order associated Volterra kernel for deriving two algorithms that allow to estimate the parameters of the linear part of these channels. In the case of a Wiener channel, the associated Volterra kernel is a tensor satisfying a rank-one PARAFAC decomposition whose the parameters can be estimated by means of an alternating least squares (ALS) algorithm. In the case of a Hammerstein channel, its associated Volterra kernel is a diagonal tensor, which leads to a closed-form solution for estimating the parameters of the linear block. The coefficients of the nonlinear block modeled as a fifth degree polynomial are then estimated by means of the standard non recursive least squares (LS) algorithm. The performance of the proposed identification methods is illustrated by means of Monte Carlo simulation results.
\end{abstract}

\section{Keywords}

Wiener and Hammerstein models, Volterra kernels, PARAFAC decomposition, Channel estimation, ALS algorithm.

\section{INTRODUCTION}

Block-structured nonlinear (NL) models are very used for modeling nonlinear dynamical systems [9]. These models are generally constituted by a cascade of time-invariant linear dynamic blocks and nonlinear static blocks approximated by polynomials of finite degree. Wiener and Hammerstein models are two well-known cases of such block-structured NL models. A Wiener model consists of a finite impulse response (FIR) linear subsystem followed by a static nonlinear subsystem. A Hammerstein model is the reverse of a Wiener model, i.e., a static nonlinear block followed by a FIR linear block. These models play an important role in various fields of application including physiological and biological systems [13, 15] and digital communication systems which utilize high-power amplifiers as used in satellites or base stations [8, 1].

Several methods have been proposed for identifying Wiener and Hammerstein models. Recursive algorithms are proposed in
[14, 19]. Iterative algorithms have also been developed for Wiener systems [21]. Some works consider subspace-based algorithms for the identification of the linear and nonlinear parts of Hammerstein [16] and Wiener systems [4].

In this paper, we propose two methods for identifying fifth-order Wiener and Hammerstein systems. These methods estimate separately and sequentially the linear dynamic block and the nonlinear static block under the form of a fifth degree polynomial. For estimating the linear subsystem, we exploit the special form of the fifth-order associated Volterra kernel. Volterra kernels can be viewed as tensors [6], and they can be estimated using i.i.d. inputs [12]. In the case of a Wiener system, the associated Volterra kernel is a tensor satisfying a rank-one PARAFAC decomposition whose the parameters can be estimated by means of an alternating least squares (ALS) algorithm [10]. In the case of a Hammerstein system, its associated Volterra kernel is a diagonal tensor, which leads to a closed-form solution for estimating its parameters. The coefficients of the nonlinear block modeled as a fifth degree polynomial are then estimated by means of the standard non recursive least squares (LS) algorithm. The proposed estimation methods generalize the results of [5, 7] which use the third-order associated Volterra kernel instead of the fifth-order one.

The rest of this paper is organized as follows. Section II describes the nonlinear Wiener and Hammerstein models and recalls the expression of associated Volterra kernels. In Section III, we present tensor-based approaches for identifying Wiener and Hammerstein systems. The proposed identification methods are illustrated by means of some Monte Carlo simulation results in Section IV, before concluding the paper in Section V.

Notations : Scalars, vectors, matrices and hight-order tensors are written as lower-case $(a, b, \cdots)$, bold lower-case $(\mathbf{a}, \mathbf{b}, \cdots)$, bold upper-case $(\mathbf{A}, \mathbf{B}, \cdots)$ and blackboard $(\mathbb{A}, \mathbb{B}, \cdots)$ letters, respectively. $\mathbf{A}^{T}, \mathbf{A}^{H}, \mathbf{A}^{*} \mathbf{A}^{+}$denote transpose, transconjugate (Hermitian transpose), complex conjugate, and Moore-Penrose pseudoinverse of $\mathbf{A}$, respectively. The vector $\mathbf{A}_{i .}$ (resp. $\mathbf{A}_{. j}$ ) represent the $i^{t h}$ row (resp. $j^{t h}$ column) of $\mathbf{A}$. The scalars $a_{i}, a_{i, j}$ and $a_{i_{1}, \cdots, i_{N}}$ denote the $i^{\text {th }}$ element of $\mathbf{a}$, the $(i, j)^{t h}$ element of $\mathbf{A}$ and the 


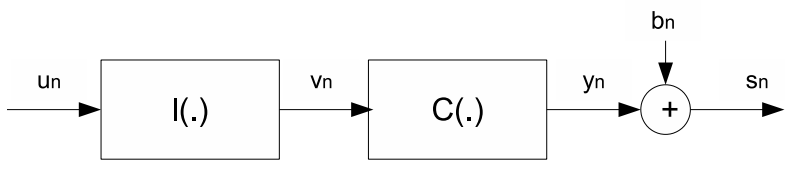

Fig. 1. Wiener model.

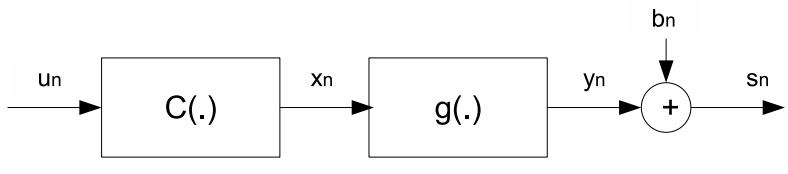

Fig. 2. Hammerstein model.

$\left(i_{1}, \cdots, i_{N}\right)^{t h}$ element of $\mathbb{A}$, respectively. $\otimes$ denotes the Kronecker product.

\section{WIENER AND HAMMERSTEIN MODELS AND THEIR ASSOCIATED VOLTERRA KERNELS}

Let us consider single input single output (SISO) nonlinear communication channels of Wiener and Hammerstein types depicted in Figures 1 and 2 respectively. We denote by $u_{n}, y_{n}, v_{n}$ and $x_{n}$, the input, the output, and intermediate signals, respectively. An additive noise signal $b_{n}$ is present at the output of the system : $s_{n}=y_{n}+b_{n}$. These models are composed of a linear filter with impulse response $l($.$) and g($.$) , of memory M$, and a memoryless nonlinear subsystem $C$ (.) that represents a high power amplifier (HPA). It has been shown that the equivalent baseband representation of the nonlinear part contains only odd-order terms [20 17]. The output of the Wiener model is described by means of the following equations :

$$
\begin{aligned}
y_{n} & =C\left(v_{n}\right)=\sum_{p=0}^{P} c_{2 p+1}\left[v_{n}\right]^{p+1}\left[v_{n}^{*}\right]^{p} \\
& =v_{n} \sum_{p=0}^{P} c_{2 p+1}\left|v_{n}\right|^{2 p}
\end{aligned}
$$

where $v_{n}=\sum_{i=0}^{M-1} l_{i} u_{n-i}$. The input-output relationship, in baseband, can be rewritten as the following equivalent Volterra model [11] :

$$
\begin{array}{r}
y_{n}=\sum_{p=0}^{P} \sum_{i_{1}, \cdots, i_{2 p+1}}^{M-1} h_{2 p+1}\left(i_{1}, \cdots, i_{2 p+1}\right) \\
\times \prod_{k=1}^{p+1} u_{n-i_{k}} \prod_{k=p+2}^{2 p+1} u_{n-i_{k}}^{*}
\end{array}
$$

where $h_{2 p+1}\left(i_{1}, \cdots, i_{2 p+1}\right)$ denotes the $(2 p+1)^{t h}$ order Volterra kernel given by :

$$
h_{2 p+1}\left(i_{1}, \cdots, i_{2 p+1}\right)=c_{2 p+1} \prod_{k=1}^{p+1} l_{i_{k}} \prod_{k=p+2}^{2 p+1} l_{i_{k}}^{*}
$$

For a Hammerstein model, the output signal is given by :

$$
\begin{aligned}
y_{n} & =\sum_{i=0}^{M-1} g_{i} x_{n-i}=\sum_{i=0}^{M-1} g_{i} C\left(u_{n-i}\right) \\
& =\sum_{i=0}^{M-1} g_{i} \sum_{p=0}^{P} c_{2 p+1} u_{n-i}\left|u_{n-i}\right|^{2 p} \\
& =\sum_{p=0}^{P} c_{2 p+1} \sum_{i=0}^{M-1} g_{i} u_{n-i}\left|u_{n-i}\right|^{2 p},
\end{aligned}
$$

The $(2 p+1)^{t h}$ order Volterra kernel associated with the Hammerstein model is given by :

$$
h_{2 p+1}\left(i_{1}, \cdots, i_{2 p+1}\right)=c_{2 p+1} g_{i} \prod_{k=1}^{2 p+1} \delta_{i_{k}, i}
$$

where $\delta_{i_{k}, i}$ is the Kronecker delta. So, we can conclude that the Volterra kernel associated with the Wiener model is a tensor admiting a rank-one PARAFAC decomposition, while it is diagonal for the Hammerstein model.

\section{TENSOR-BASED METHODS FOR WIENER AND HAMMERSTEIN SYSTEMS IDENTIFICATION}

In this section, we present two methods for identifying Wiener and Hammerstein models. These methods are composed of three basic steps :

(1) Estimate the fifth-order Volterra kernel associated with the system to be identified using input-output measurements obtained with i.i.d. inputs [12].

(2) Estimate the parameters of the linear subsystem using the estimated Volterra kernel in step (1).

(3) Estimate the parameters of the nonlinear subsystem using the non recursive LS algorithm.

\subsection{Estimation of Volterra kernels associated with Wiener and Hammerstein models}

In this paper, the fifth-order Volterra kernel associated with the fifth-order Wiener and Hammerstein models is estimated by means of a closed-form expression as described in [12]. This closed-form expression has been derived under the following assumptions :

- The input signal is real-valued, zero-mean, i.i.d. with a symmetrical probability distribution function.

- The input/output signals are ergodic and stationary at least up to the sixth-order.

- The additive noise is zero-mean and independent of the input signal.

\subsection{Tensor-based approaches for Wiener model identification}

3.2.1 Estimation of the linear subsystem. From Eq. (5), the fifthorder Volterra kernel is given by :

$$
h_{5}\left(i_{1}, i_{2}, i_{3}, i_{4}, i_{5}\right)=c_{5} l_{i_{1}} l_{i_{2}} l_{i_{3}} l_{i_{4}}^{*} l_{i_{5}}^{*} .
$$

It can be seen as a tensor $\mathbb{H}_{5} \in \mathcal{C}^{M \times M \times M \times M \times M}$ satisfying a rankone PARAFAC decomposition. Define the vector of impulse response coefficients of the linear subsystem $\mathbf{l}=\left[l_{0} \cdots l_{M-1}\right]^{T}$, with $l_{0}=1$, a standard constraint to guarantee model uniqueness. Now, 
Table 1. Single-step ALS algorithm

Form the unfolded matrix $\mathbf{H}_{1}$ of the estimated fifth-order Volterra kernel associated with the Wiener system

(1) $k=0$, initialize $\hat{\mathbf{l}}_{0}$ with random values,

(2) $k=k+1$,

(3) Compute $\hat{\mathbf{l}}_{k}=\left[\hat{\mathbf{g}}_{k-1}^{+} \mathbf{H}_{1}\right]^{T}$, with $\hat{\mathbf{g}}_{k-1}=\hat{\mathbf{l}}_{k-1} \otimes \hat{\mathbf{l}}_{k-1} \otimes$ $\hat{\mathbf{l}}_{k-1}^{*} \otimes \hat{\mathbf{l}}_{k-1}^{*}$,

(4) Return to step (2) until a stop criterion is reached,

(5) Normalize $\hat{\mathbf{l}}$ by dividing its components by the first entry $\hat{\mathbf{l}}_{k}(1)$.

define the vector factors associated with this PARAFAC decomposition as :

$$
\mathbf{a}=\mathbf{b}=\mathbf{c}=\mathbf{l}, \quad \mathbf{d}=\mathbf{l}^{*}, \quad \mathbf{e}=c_{5} \mathbf{l}^{*}
$$

The mode-1 matrix unfolding of $\mathbb{H}_{5}$, denoted $\mathbf{H}_{1} \in \mathcal{C}^{M^{4} \times M}$ is given by :

$$
\mathbf{H}_{1}=(\mathbf{b} \otimes \mathbf{c} \otimes \mathbf{d} \otimes \mathbf{e}) \mathbf{a}^{T}=c_{5}\left(\mathbf{l} \otimes \mathbf{l} \otimes \mathbf{l}^{*} \otimes \mathbf{l}^{*}\right) \mathbf{l}^{T} .
$$

From this equation, we can build an iterative LS algorithm which computes an updated estimate $\hat{\mathbf{l}}_{k}$ at iteration $k$ from the estimated value $\hat{\mathbf{l}}_{k-1}$ calculated at iteration $k-1$, by means of the following equation

$$
\hat{\mathbf{l}}_{k}=\left[\left(\hat{\mathbf{l}}_{k-1} \otimes \hat{\mathbf{l}}_{k-1} \otimes \hat{\mathbf{l}}_{k-1}^{*} \otimes \hat{\mathbf{l}}_{k-1}^{*}\right)^{+} \mathbf{H}_{1}\right]^{T}
$$

The corresponding iterative LS algorithm for estimating the linear block of a Wiener system is summarized in Table 1 .

3.2.2 Estimation of the nonlinear part. Define the following filtered input sequence :

$$
\bar{u}_{n}^{(2 p+1)}=\sum_{i_{1}, \cdots, i_{2 p+1}=0}^{M-1} \prod_{k=1}^{p+1} \hat{l}_{i_{k}} u_{n-i_{k}} \prod_{k=p+2}^{2 p+1} \hat{l}_{i_{k}}^{*} u_{n-i_{k}}^{*}
$$

where $\hat{l}_{i_{k}}$ is the estimated value of $l_{i_{k}}$ obtained at convergence of the Algorithm in Table 1. Then, the output signal reconstructed using the estimated linear block can be written as :

$$
\hat{y}_{n}=\overline{\mathbf{u}}_{n}^{T} \mathbf{c}
$$

where $\mathbf{c}=\left(\begin{array}{llll}c_{1} & \cdots & c_{2 P+1}\end{array}\right)^{T}$ and $\overline{\mathbf{u}}_{n}=\left(\begin{array}{lll}\bar{u}_{n}^{(1)} & \cdots & \bar{u}_{n}^{(2 P+1)}\end{array}\right)^{T}$. By concatenating the output measurements for $n=1, \cdots, N$, the LS estimate of the polynomial coefficients of the nonlinear subsystem is given by :

$$
\hat{\mathbf{c}}=\mathbf{U}^{+} \hat{\mathbf{y}}
$$

where $\mathbf{U}=\left(\overline{\mathbf{u}}_{1} \cdots \overline{\mathbf{u}}_{N}\right)^{T}$, and $\hat{\mathbf{y}}=\left(\hat{y}_{1} \cdots \hat{y}_{N}\right)^{T}$.

\subsection{Tensor-based approach for Hammerstein model identification}

3.3.1 Estimation of the linear subsystem. From (9), the fifthorder Volterra kernel associated with a Hammerstein system is given by :

$$
h_{5}\left(i_{1}, i_{2}, i_{3}, i_{4}, i_{5}\right)= \begin{cases}c_{5} g_{i}, \text { if } i_{1}=i_{2}=\cdots=i_{5}=i \\ 0, \quad \text { else. }\end{cases}
$$

It is a diagonal tensor. Assuming $g_{0}=1$, we can deduce that $c_{5}=$ $h_{5}(0,0,0,0,0)$. The parameters of the linear subsystem can then be estimated using the following closed-form equation

$$
\hat{g}_{i}=\frac{h_{5}(i, i, i, i, i)}{h_{5}(0,0,0,0,0)}, i=1, \cdots, M-1 .
$$

3.3.2 Estimation of the nonlinear block. Define the following filtered input sequence

$$
\bar{u}_{n}^{(2 p+1)}=\sum_{i=0}^{M-1} \hat{g}_{i} u_{n-i}\left|u_{n-i}\right|^{2 p},
$$

The reconstructed output signal and the LS estimate of the polynomial coefficients of the nonlinear block can be calculated by means of the same equations $(15)$ and $(16)$ as for the Wiener model.

\section{SIMULATIONS RESULTS}

In this section, we present some Monte Carlo simulation results for illustrating the performance of the proposed identification methods.

The performance criteria were calculated by averaging the results over $N_{s}=300$ simulated Wiener or Hammerstein models whose parameters were randomly drawn from a Gaussian distribution. For each model of memory $M=3$ and nonlinearity degree $P=5$, the generated output signal was perturbed by $N_{b}=10$ different additive, zero-mean, white Gaussian noise sequences. The performance is evaluated in terms of Normalized Mean Square Error (NMSE) on the estimated fifth-order Volterra kernel associated with each simulated model $\left(\mathrm{NMSE}_{h_{5}}\right)$, the output signal $\left(\mathrm{NMSE}_{s}\right)$, and the estimated parameters of the linear subsystem $\left(\mathrm{NMSE}_{l}\right)$ and of the nonlinear block $\left(\mathrm{NMSE}_{c}\right)$.

$$
\begin{aligned}
& \operatorname{NMSE}_{h_{5}}=10 \log \left(\frac{1}{N_{s} N_{b}} \sum_{m=1}^{N_{s}} \sum_{b=1}^{N_{b}} \frac{\left\|\hat{\mathbb{H}}_{m, b}-\mathbb{H}_{m}\right\|_{F}^{2}}{\left\|\mathbb{H}_{m}\right\|_{F}^{2}}\right), \\
& \mathrm{NMSE}_{s}=10 \log \left(\frac{1}{N_{s} N_{b}} \sum_{m=1}^{N_{s}} \sum_{b=1}^{N_{b}} \frac{\left\|\hat{\mathbf{s}}_{m, b}-\mathbf{s}_{m}\right\|_{2}^{2}}{\left\|\mathbf{s}_{m}\right\|_{2}^{2}}\right), \\
& \mathrm{NMSE}_{l}=10 \log \left(\frac{1}{N_{s} N_{b}} \sum_{m=1}^{N_{s}} \sum_{b=1}^{N_{b}} \frac{\left\|\hat{\theta}_{m, b}-\theta_{m}\right\|_{2}^{2}}{\left\|\theta_{m}\right\|_{2}^{2}}\right), \\
& \mathrm{NMSE}_{c}=10 \log \left(\frac{1}{N_{s} N_{b}} \sum_{m=1}^{N_{s}} \sum_{b=1}^{N_{b}} \frac{\left\|\hat{\mathbf{c}}_{m, b}-\mathbf{c}_{m}\right\|_{2}^{2}}{\left\|\mathbf{c}_{m}\right\|_{2}^{2}}\right),
\end{aligned}
$$

where $\mathbf{s}_{m}, \hat{\mathbf{s}}_{m, b}$, and $\hat{\mathbb{H}}_{m, b}$ denote, respectively, the simulated output vector, the output vector reconstructed using the estimated parameters $\left(\hat{\theta}_{m, b}, \hat{\mathbf{c}}_{m, b}\right)$, and the fifth-order Volterra kernel reconstructed from the estimated Wiener $\left(\hat{\theta}_{m, b}=\hat{\mathbf{l}}_{m, b}\right)$ or Hammerstein $\left(\hat{\theta}_{m, b}=\hat{\mathbf{g}}_{m, b}\right)$ model, associated with the $m^{t h}$ simulated model and $b^{\text {th }}$ additive noise sequence. The input sequence is an i.i.d. 6-RMS (Random Multilevel Sequence), with six levels $\{ \pm 1, \pm 2 / 3, \pm 1 / 3\}$, and length $N$. To guarantee the i.i.d. property, the input sequence was simulated according to the method described in [18].

\subsection{Estimation of Wiener models}

Figure 3 shows the different NMSEs obtained with an input length $N=6480$, while Figure 4 illustrates the impact of SNR, length $N$ 


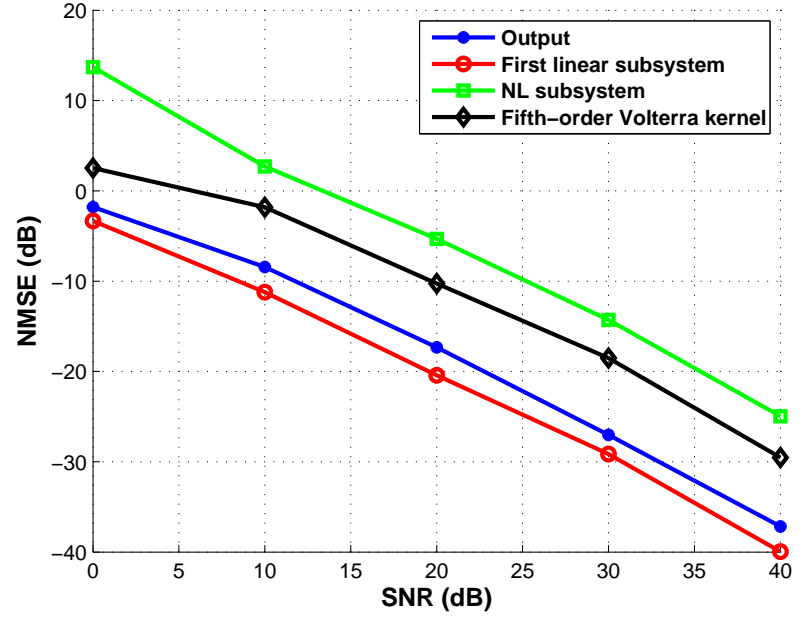

Fig. 3. NMSEs for different values of SNR for Wiener models

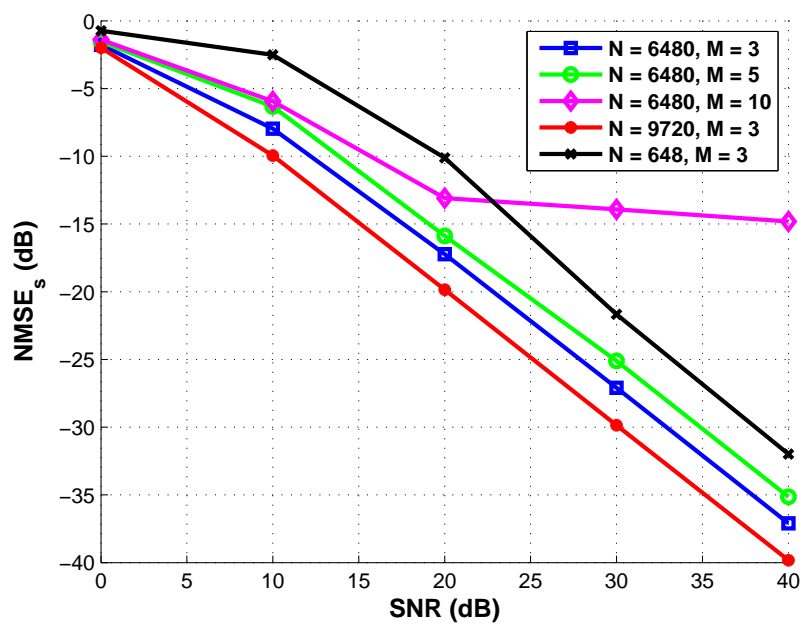

Fig. 4. Impact of SNR, $N$ and $M$ on $\mathrm{NMSE}_{s}$ for Wiener models

and memory $M$ on the $\mathrm{NMSE}_{s}$. From these simulation results, as expected, the different NMSE decrease when the SNR and/or the input-output measurements number $(N)$ increase. On the contrary, the $\mathrm{NMSE}_{s}$ increases when the memory length $(M)$ increases, which corresponds to an increase of the number of parameters to be estimated. Finally, we have to note that the convergence of the ALS algorithm which takes symmetry of the Volterra kernel into account, is very sensitive to the initialization. So, for improving the performance, a multi-initialization scheme is employed, that is, different initializations are used and the one giving the best $\mathrm{NMSE}_{s}$ is kept.

\subsection{Estimation of Hammerstein models}

Table 2 provides the mean value and the standard deviation of the estimated parameters, both for the linear and the nonlinear blocks, for three different values of SNR. From these results, we can conclude that the estimated parameters of the two subsystems are
Table 2. Mean value and standard deviation of the estimated parameters of the two subsystems of Hammerstein system

\begin{tabular}{|c|c|c|c|}
\hline \multirow{2}{*}{$\begin{array}{l}\text { Actual } \\
\text { Parameters } g(.)\end{array}$} & \multicolumn{3}{|c|}{ Estimated Parameters $\hat{g}()}$. \\
\hline & $\mathrm{SNR}=0 \mathrm{~dB}$ & $\mathrm{SNR}=20 \mathrm{~dB}$ & $\mathrm{SNR}=40 \mathrm{~dB}$ \\
\hline 1 & 1 & 1 & 1 \\
\hline$-0.9858+0.3643 j$ & $\begin{array}{l}-0.8170-0.0223 j \\
\pm 1.0074\end{array}$ & $\begin{array}{l}-0.9905+0.3551 j \\
\pm 0.0174\end{array}$ & $\begin{array}{l}-0.9872+0.3639 j \\
\pm 0.0016\end{array}$ \\
\hline $1.5749+0.1461 j$ & $\begin{array}{l}0.9395+0.6020 j \\
\pm 1.1373\end{array}$ & $\begin{array}{l}1.5663+0.1496 j \\
\pm 0.0690\end{array}$ & $\begin{array}{l}1.5763+0.1478 j \\
\pm 0.0018\end{array}$ \\
\hline
\end{tabular}

\begin{tabular}{|l||l|l|l|}
\hline \multirow{2}{*}{ Actual } & \multicolumn{3}{|c|}{ Estimated Parameters $\hat{c}()}$. \\
\cline { 2 - 4 } Parameters $c()$. & SNR=0dB & SNR=20dB & SNR=40dB \\
\hline \multirow{2}{*}{$-0.5182+0.8955 j$} & $-0.2917+0.6037 j$ & $-0.5178+0.8946 j$ & $-0.5175+0.8951 j$ \\
& \pm 0.8474 & \pm 0.0110 & \pm 0.0017 \\
$-0.3523+0.0592 j$ & $-0.8175+0.4592 j$ & $-0.3618+0.0926 j$ & $-0.3521+0.0616 j$ \\
& \pm 2.3832 & \pm 0.1714 & \pm 0.0132 \\
$-0.7817-1.1243 j$ & $-0.9181-0.5130 j$ & $-0.7866-1.1242 j$ & $-0.7802-1.1228 j$ \\
& \pm 1.3983 & \pm 0.0734 & \pm 0.0064 \\
$0.5146+2.2071 j$ & $0.8538+1.3205 j$ & $0.5171+2.1824 j$ & $0.5158+2.2032 j$ \\
& \pm 2.3601 & \pm 0.0395 & \pm 0.0065 \\
$1.2132-1.0222 j$ & $1.1216-1.0222 j$ & $1.2203-1.0222 j$ & $1.2103-1.0222 j$ \\
& \pm 1.0992 & \pm 0.0434 & \pm 0.0015 \\
\hline
\end{tabular}

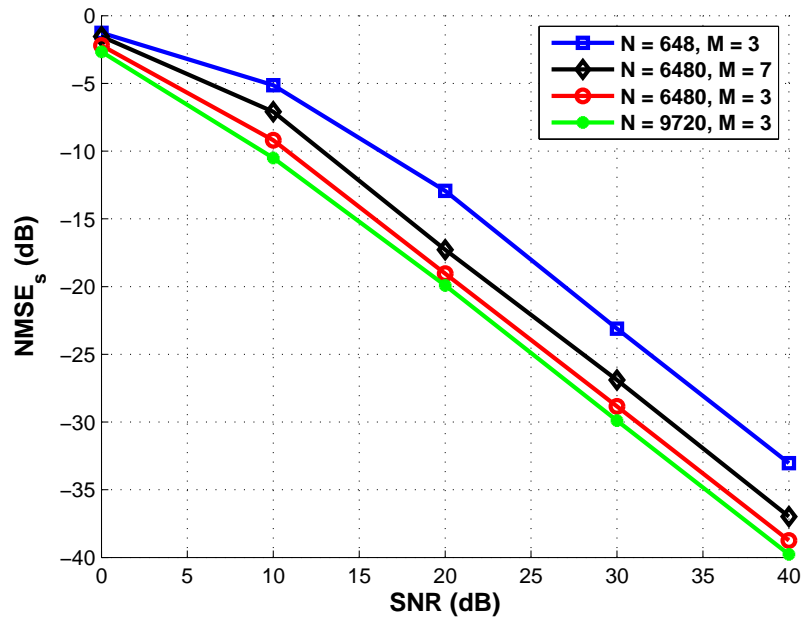

Fig. 5. Impact of SNR, $N$ and $M$ on $\mathrm{NMSE}_{s}$ for Hammerstein models

close to the actual ones for $\mathrm{SNR} \geq 20 d B$. However, for $\mathrm{SNR}=0 \mathrm{~dB}$, the estimation performance is notably degraded, showing that the closed-form solution (18) is not robust to the additive noise.

The impact of SNR, $N$, and $M$ on $\mathrm{NMSe}_{s}$ is shown in Figure 5 As previously, the $\mathrm{NMSE}_{s}$ decreases when the SNR and/or the number $N$ of measurements increase, while it increases when $M$ increases.

\section{CONCLUSION}

We have presented two methods for identifying fifth-order Wiener and Hammerstein nonlinear communication channels. These methods rely on the associated fifth-order Volterra kernel, estimated by means of a closed-form solution which uses input-output cross- 
moments, with an i.i.d. input signal [12]. They estimate separately and sequentially the linear and the nonlinear blocks. The parameters of the linear block are directly linked with the Volterra kernel. This kernel viewed as a complex symmetric tensor admits a rank-one PARAFAC decomposition in the case of Wiener systems, and it is diagonal in the case of Hammerstein systems. These special tensor structures are exploited for deriving a single step ALS algorithm with multi random initializations, in the first case, and a very simple closed-form solution in the second case. The nonlinear block is then estimated using a standard non-recursive LS algorithm. As illustrated by means of simulation results, the proposed estimation methods provide very good performance for a wide range of SNR values. As perspective for future work, we can mention the study of the statistical efficiency of the proposed estimators through the determination of Cramér-Rao bounds (CRB), as recently carried out for tensor PARAFAC decompositions with structured factor matrices [2]. Another perspective concerns the comparison of the proposed channel estimation methods with a method based on Volterra-PARAFAC modeling of nonlinear channels [3].

\section{REFERENCES}

[1] N.J. Bershad, P. Celka, and S. Mclaughlin. Analysis of stochastic gradient identification of Wiener-Hammerstein systems for nonlinearities with Hermite polynomial expansions. IEEE Trans. on Signal Processing, 49(5) :1060-1072, May 2001.

[2] M. Boizard, J.H. Goulart, R. Boyer, G. Favier, and P. Comon. Statistical efficiency of structured CPD estimation applied to Wiener-Hammerstein modeling. Proc. European Signal Process. Conf. (EUSIPCO), Nice, France, Aug 2015.

[3] T. Bouilloc and G. Favier. Nonlinear channel modeling and identification using bandpass Volterra-PARAFAC models. Signal Processing, Elsevier, 92(6) :1492-1498, June 2012.

[4] X. Chen, H.T Fang, and X Wang. Subspace identification for Wiener systems with general nonlinearity. 30th Chinese Control Conference (CCC), Yantai, China, pages 1696-1701, 2011.

[5] G. Favier. Nonlinear system modeling and identification using tensor approaches. 10th International conference on Sciences and Techniques of Automatic control and computer engineering (STA'2009), Hammamet, Tunisie, Dec 2009.

[6] G. Favier, A. Kibangou, and Bouilloc T. Nonlinear system modeling and identification using Volterra-PARAFAC models. Int. J. of Adaptive Control and Sig. Proc., 26 :30-53, 2012.

[7] G. Favier and A. Y. Kibangou. Tensor-based methods for system identification. 9th International conference on Sciences and Techniques of Automatic control and computer engineering (STA'2008), Sousse, Tunisie, 3(1) :840-869, Dec 2008.

[8] X.N. Fernando and A.B. Sesay. Fiber wireless channel estimation using correlation properties of PN sequences. $\mathrm{Ca}$ nadian Journal of Electrical and Computer Enginneering, 26(2), April 2001.

[9] R. Haber and L. Keviczky. Nonlinear system identification Input-output modeling approach, vol. 1 of Mathematical modelling : theory and applications. Kluwer Academy Publishers, 1999.

[10] R. A. Harshman. Foundations of the PARAFAC procedure : Model and conditions for an "explanatory" multi-mode factor analysis. UCLA Working Papers in Phonetics, 16 :1-84, Dec. 1970.

[11] A. Y. Kibangou and G. Favier. Matrix and tensor decompositions for identification of block-structured nonlinear channels in digital transmission systems. Signal Processing Advances in Wireless Communications (SPAWC), Recife, Brazil, pages 281-285, July 2008.

[12] A. Y. Kibangou and G. Favier. Identification of fifth-order Volterra systems using i.i.d. inputs. IET Signal Processing, 4(1) :30-44, Feb 2010.

[13] M.J. Korenberg and I.W. Hunter. The identification of nonlinear biological systems : LNL cascade models. Biological Cybernetics, pages 125-134, 1986.

[14] F. Le, I. Markovsky, C. Freeman, and E. Rogers. Recursive identification of Hammerstein structure. In, 18th IFAC World Congress, Milan, Italy, August 28 - September 2011.

[15] V. Z. Marmarelis. Nonlinear dynamic modeling of physiological systems. IEEE Press, John Wiley \& Sons, 2004.

[16] M. Pouliquen, F. Giri, O. Gehan, E. Pigeon, M. Frikel, and B Targui. Subspace identification for Hammerstein systems with nonparametric input backlash and switch nonlinearities. Conference on Decision and Control, Firenze, Italy, pages 4302-4307, 2013.

[17] R. Raich. Nonlinear System Identification and Analysis with Applications to Power Amplifier Modeling and Power Amplifier Predistortion. $\mathrm{PhD}$ thesis, School of Electrical and Computer Engineering Georgia Institute of Technology, 2004.

[18] C.H. Tseng and E.J. Powers. Identification of Nonlinear Channels in Digital Transmission Systems. Proc. IEEE Signal Processing Workshop on Higher-order Statistics, South Lake Tahoe, CA, pages 42-45, June 1993.

[19] J. Voros. Recursive identification of Wiener system with two-segment polynomial nonlinearities. J. Electr. Engin, 59(1) :40-44, 2008.

[20] G.T. Zhou and R. Raich. Spectral analysis of polynomial nonlinearity with applications to RF power amplifiers. EURASIP Journal on Applied Signal Processing, 12 :1831-1840, 2004.

[21] L. Zhou, X. Li, and F. Pan. Least-squares-based iterative identification algorithm for Wiener nonlinear systems. Journal of Applied Mathematics, 2013 :1-6, 2013. 